\title{
Healthcare Quality and Hospital Financial Performance: A Multilevel Framework
}

\author{
Subhajit Chakraborty \\ Department of Management and Decision Sciences, \\ Coastal Carolina University, Conway, South Carolina 29528 \\ Email: schakrabo@ coastal.edu
}

\begin{abstract}
Given the recent reports on much-needed healthcare quality improvement in the U.S. we suggest that it is now time to adopt a systemic perspective to measuring quality in U.S. healthcare and tracking it closely to hospital financial performance. Using Donabedian's (1966, 1968, 1988) Structure-Process-Outcome (SPO) classification we attempt to offer a multi-level conceptual framework tying in the antecedents to and outcomes of patient care quality that can be used by U.S. hospitals to improve the quality of care available to patients and ensure their own viability over the long run. We also address the measurement challenges for a future empirical measurement and conclude with implications for healthcare research and the medical practitioners and U.S. hospitals.
\end{abstract}

Keywords: patient care quality, multi-level framework, hospital financial performance

\section{INTRODUCTION}

According to a recent Commonwealth Fund reportMirror, Mirror on the Wall: 2014 Update (Davis et al., 2014), despite years of multi-disciplinary research on healthcare quality improvement, the United States ranked last overall among 11 industrialized countries on systemic measures of health quality, efficiency, access to care, equity, and healthy lives. The U.S. had the highest costs but lowest performance; on an average, the U.S. spent $\$ 8,508$ per person on health care in 2011, compared with $\$ 3,406$ in the United Kingdom, which ranked first overall. Many journal papers, newspaper articles, blogs and reports have been indicating that the U.S. needs to systematically improve its healthcare quality performance (Becker and Gamble, 2015; Boyer and Pronovost, 2010).

While a lot of detailed quality metrics based on the nature of the ailment (such as stroke, pneumonia, cardiac arrest), the number of days of hospitalization necessary for the treatment, hospital readmission rates and mortality rates have now become popular as standardized metrics to measure healthcare quality for public reporting (CMS, 2016), patient safety has emerged as the key aspect of healthcare quality (Kohn et al., 1999; Pronovost et al., 2006). This is evident in several studies across various fields such as nursing (Laschinger and Leiter, 2006; Rogers et al., 2004), healthcare (Pronovost et al., 2006; Singer et al., 2007), operations management (OM) (Chandrasekaran et al., 2012; McFadden et al., 2009) and strategic management (Douglas and Ryman, 2003; Huckman and Zinner, 2008). Patient safety, however, is only a component of one of the dimensions of patient care quality (PCQ) which has been labeled technical quality (Dagger et al., 2007). Recent studies on quality of patient care imply that there are three more aspects of PCQ such as interpersonal, environmental and administrative quality (Chang et al., 2009; Dagger et al., 2007).

Aside from an extensive research on medicine and disease-specific cures, extant healthcare literature does not offer a comprehensive framework for measuring patient care quality in an integrated manner. The objective of this research is to offer an integrated conceptual framework that ties in all the antecedents to, and outcomes of PCQ which can be used by U.S. hospitals to achieve twin objectives: improve the quality of care available to their patients; and help hospitals sustain and thrive over the long run. We use Quality Management (QM) (Feigenbaum, 1961) as the underlying theory to justify the propositions and draw from Donabedian's (1966) Structure-Process-Outcome (SPO) classification to aid our framework. In so doing we attempt to address the question: what are the antecedents to, and outcomes of patient care quality in the U.S. hospital ecosystem?

Quality management (QM) is an integrative philosophy of management for continuously improving the quality of products and processes (Feigenbaum, 1961). The basic premise is that the quality of products/services and processes of their manufacture/generation is the responsibility of everyone who is involved with the creation or consumption of the products (Feigenbaum, 1961). QM highlights the involvement of management, workforce, suppliers, and even customers in order to meet or exceed customer expectations (Deming, 1986; Kaynak and Hartley, 2008). We draw from tenets of QM philosophy to justify our propositions.

According to the Donabedian's SPO model (1966, $1968,1988)$ patient care quality information can be drawn from three categories: structure, process, and outcomes. Structure describes the context of care, i.e., includes hospital buildings, staff, financing, and equipment. Process denotes the transactions between patients and healthcare providers during the medical treatment and beyond. Outcomes refer to the effects of healthcare on the health status of patients and populations. The SPO model is being widely used in healthcare literature as a foundational classification to build other frameworks (Jolley et al., 2017; Voyce et al., 2015).

This paper is structured as follows. Following an extensive review of extant interdisciplinary literature, we first identify the determinants and outcomes of PCQ. Then, we highlight the variables at the individual patient level in a 
U.S. hospital. Third, we discuss the healthcare team-level antecedents. Next, we explicate the hospital-level outcomes. Fifth, drawing from QM philosophy we suggest a conceptual overarching framework and use Donabedian's SPO guidelines $(1966 ; 1968 ; 1988)$ to place our framework in perspective. We then offer propositions about expected relationships among the variables. Finally, we discuss the implications of our framework, note the limitations and conclude with future directions.

\section{LITERATURE REVIEW}

We conducted an extensive review of interdisciplinary literature comprising articles published in healthcare, human resources management, medicine, marketing, nursing, organizational behavior, operations management (OM), and strategic management areas; and several newspapers and magazines, online blogs and reports. From this review, we identified the quality-related variables that affect patients' medical treatment in a U.S. hospital on one hand, and hospital sustenance, on the other. Then, drawing from the tenets of QM philosophy (Feigenbaum, 1961) we first grouped the variables into three categories: individual patient-centric: PCQ, medical treatment expenses and patient satisfaction; healthcare team-related antecedents: healthcare team cohesion and healthcare team effectiveness; and hospital-level outcomes: hospital reputation and hospital financial performance.

\subsection{Patient-Centric Care Quality and Satisfaction}

We define Patient care quality (PCQ) as the excellence of the medical care received by admitted patients in hospitals (Chang et al., 2009; Nelson and Niederberger, 1990). Based on a synthesis of the multi-dimensional nature of patient care quality discussed in extant literature (Dagger et al., 2007; Gill and White, 2009) it is suggested that PCQ has the following four primary dimensions: interpersonal, technical, environmental and administrative quality. Interpersonal quality reflects the relationship developed and the dyadic interplay that occurs between the healthcare team and the patient (Dagger et al., 2007; Gill and White, 2009). It takes into consideration issues such as whether healthcare teams consider their patients with respect, healthcare team members listen to what patients have to say, members give personalized attention to patients and whether team members are willing to answer questions that the patient or their kin may have. Technical quality reflects the expertise, professionalism, and competency of the healthcare team in delivering the cure (Dagger et al., 2007; Sweeney et al., 2015). It is concerned with whether patients are administered the correct medical care that is required to cure their ailment, tests (e.g., X-rays and lab tests) are ordered on patients only when required, healthcare team members are qualified, and whether they carry out their tasks competently (Dagger et al., 2007). Environmental quality comprises elements of the hospital atmosphere such as cleanliness and order and tangibles like hospital bed and required equipment for patient health needs (Dagger et al., 2007; McColl-Kennedy et al., 2017). It takes into account whether the design of the hospital is patient friendly, the lighting at the hospital is appropriate, the temperature at the hospital is pleasant and whether the furniture at the hospital is comfortable. Administrative quality facilitates the production of the core medical cure while adding value to the patient (Dagger et al., 2007; Mosadeghrad, 2013). Considerations such as whether the internal hospital services (e.g., pathology) work well, waiting time at the hospital is minimum, the hospital provides patients with a range of patient support services and whether the hospital records and documentation (e.g., billing) are error free are in the domain of this dimension of quality.

Next, we discuss two other key patient-centric variables related to PCQ. We refer to the medical treatment expenses as the actual share of their medical treatment costs that a patient ends up paying after being advised by their health insurance. In U.S. healthcare, for most patients who have some form of health insurance coverage, their health insurance company acts as an intermediary between the patient and the hospital. Depending upon the network coverage of the hospital/physicians where the procedure was done, the patient ends up having to pay a higher or lower share of the total medical costs plus markup that the hospital bills the patient's insurance company. Healthcare, medicine and OM research have identified a few factors - both patientrelated and hospital-related-that influence medical treatment costs, and thereby the actual expenses that a patient must bear. First, research has demonstrated that adherence to medication schedules, both by the patients (at their end) and doctors/nurses (when patients come back for check-up) determine the medical expenses, especially for patients suffering from chronic problems such as diabetes and hypertension (Sokol et al., 2005), anxiety and depression (DiMatteo et al., 2000) and asthma (Bender and Rand, 2004). Second, Kujala and Lillrank (2004) suggest that firms' acceptance of QM philosophy maybe influenced by their organizational culture and a continuous reduction in variation decreases the costs and increases customer acceptance. In other words, some hospitals maybe more focused than others on providing effective treatment at a lower cost. Finally, there is a tradeoff between the cost efficiency and the experiential quality of care (Venkataraman, 2015), i.e., the more the medical treatment expenses for the patient, the less is his/her experiential dimension of quality of care.

Patient satisfaction is a defined as the extent to which a patient is content with the healthcare they received from their provider. In evaluations of healthcare quality, patient satisfaction is a performance indicator often measured in a self-reported study. In marketing and healthcare literature, there are quite a few studies that have already considered patient satisfaction with a cure and patient safety and other related quality related issues from a patient's perspective (Dagger et al., 2007; Nelson and Niederberger, 1990). Patient satisfaction has also been studied in OM literature (Queenan et al., 2011), which investigated the extent to which computerized physician order entry helps improve patient satisfaction.

\subsection{Healthcare Team-Level Antecedents}

A team is defined as "a collection of individuals who are interdependent in their tasks, who share responsibility for outcomes, who see themselves and who are seen by others as an intact social entity embedded in one or more larger social 
systems (for example, business unit or corporation), and who manage their relationships across organizational boundaries" (Cohen and Bailey, 1997). In this study, healthcare team refers to the doctors, nurses and supporting staff who work together as a group to care for admitted patients in most hospitals.

Team cohesion is defined as the tendency of a team to have complete unity of purpose while working towards a goal and to satisfy the emotional needs of its members (Carron and Brawley, 2000). It has three attributesdynamic nature, instrumental basis, and an emotional dimension (Beal et al., 2003; Lott and Lott, 1965). The dynamic nature refers to how the team gradually changes over time in its strength and form during the period from team formation to disbanding. The instrumental basis refers to how people cohere for a purpose, whether it is for a specific task or for social reasons. The emotional dimension refers to how cohesion is generally pleasing to its group members. In addition to task commitment and interpersonal attraction that members feel to the team (Lott and Lott, 1965), team members take pride in being a part of a cohesive team that performs well (Beal et al., 2003).

In the hospital context, healthcare team cohesion is defined as the collective forces that influence each member of the small healthcare team to have complete unity of purpose and form an individual attraction to the team (Carron and Brawley, 2000; Price and Mueller, 1986). Tuckman (1965) suggested that individuals, during the third stage of group formation (or norming), come to know each other well and form a unity of purpose. Individuals with access to information, support, resources and opportunities to learn and grow in their team setting would be cohesive and are likely to put their best effort towards achieving the team goals.

In an organizational decision-making context, effectiveness of an action refers to whether the action taken is right or correct (Sundstrom et al., 1990). Team effectiveness refers to how well the team is able to perform to survive, adapt, maintain itself and grow (Goodman, 1986). Team effectiveness literature (Katzenbach and Smith, 2013; McGregor, 1987) suggests that effective teams have a clear unity of purpose. Team members criticize each other frequently but collectively agree on group activity. A team's performance on three important attributes determines the degree of its effectiveness. First, the ability of the team to exploit its environment to acquire scarce resources influences the team's ability to deliver (Shipper and White, 1983). Second, effective teams have a high degree of internal team efficiency, team spirit, confidence, trust, communication and support (Sundstrom et al., 1990). Third, effective teams are able to identify output goals and assess how well they can be attained (Hall, 1980). Environmental factors such as industry characteristics influence task design, which in turn, is related to internal and external group processes and group psychosocial traits such as the norms, all of which finally determine team effectiveness (Cohen and Bailey, 1997). Team cohesiveness, leadership and the team's internal activities also influence team effectiveness in a work context (Goodman, 1986).

Healthcare team effectiveness research has elaborately discussed challenges that most healthcare teams face when they try to work effectively (Buljac-Samardzic et al., 2010; Lemieux-Charles and McGuire, 2006). An empirical study with data from chronic illness care centers established the importance of team effectiveness for quality of patient care (Shortell et al., 2004). Using data from the academic departments of family medicine a qualitative study (Delva et al., 2008) highlighted some of the challenges that teams face.

Although teamwork is one of the QM practices (Kaynak, 2003; Kaynak and Hartley, 2008), not all teams are known to be effective. Members of cohesive teams are known to get along with each other and work towards achieving the collective team goals (Yukelson et al., 1984). Such effective teams are able to perform (Beal et al., 2003; Mullen and Copper, 1994). Therefore, we included team cohesion and team effectiveness instead of teamwork in the framework.

\subsection{Hospital-Level Outcomes}

In healthcare literature, it has been argued that improving patient satisfaction can have a positive impact on a hospital's reputation and its financial performance (Hall, 2008). Research has indicated that reporting positive ratings to the public improves the collective hospital reputations (Hibbard et al., 2005). Mira et al. (2014) demonstrated that the social reputation of hospitals and the perceptions of patients or relatives of patient safety were correlated. It is also known that the relative standings of the top 50 U.S. hospitals largely reflect the subjective reputations of those hospitals (Sehgal, 2010). Adverse outcome experience, a proxy for hospital reputation, may determine how patients choose their future hospital (de Mheen et al., 2010).

Alexander and colleagues (2006) show that continuous quality improvement has a measurable impact on organizational performance. Hospitals that effectively implement quality improvement strategies can ultimately improve their financial performance. Goes and Zhan (1995) validated the hospital-physician financial integration efforts, and found that involvement of physicians in hospital governance improves its financial performance. Research has shown that there is a positive association between hospital strategic planning processes and financial performance (Kaissi and Begun, 2008). Further, hospitals with lower margins and higher costs are likely to implement strategies to integrate physicians and align physician and hospital interests (Mark et al., 1998); therefore, individual physician reputations are more likely to impact hospital reputations. Nelson and colleagues' (1992) findings suggest that measurable improvements in patients' judgments of hospital quality could improve hospitals' financial performance. In sum, hospital reputation and financial performance are key outcomes related to patient satisfaction and PCQ. 


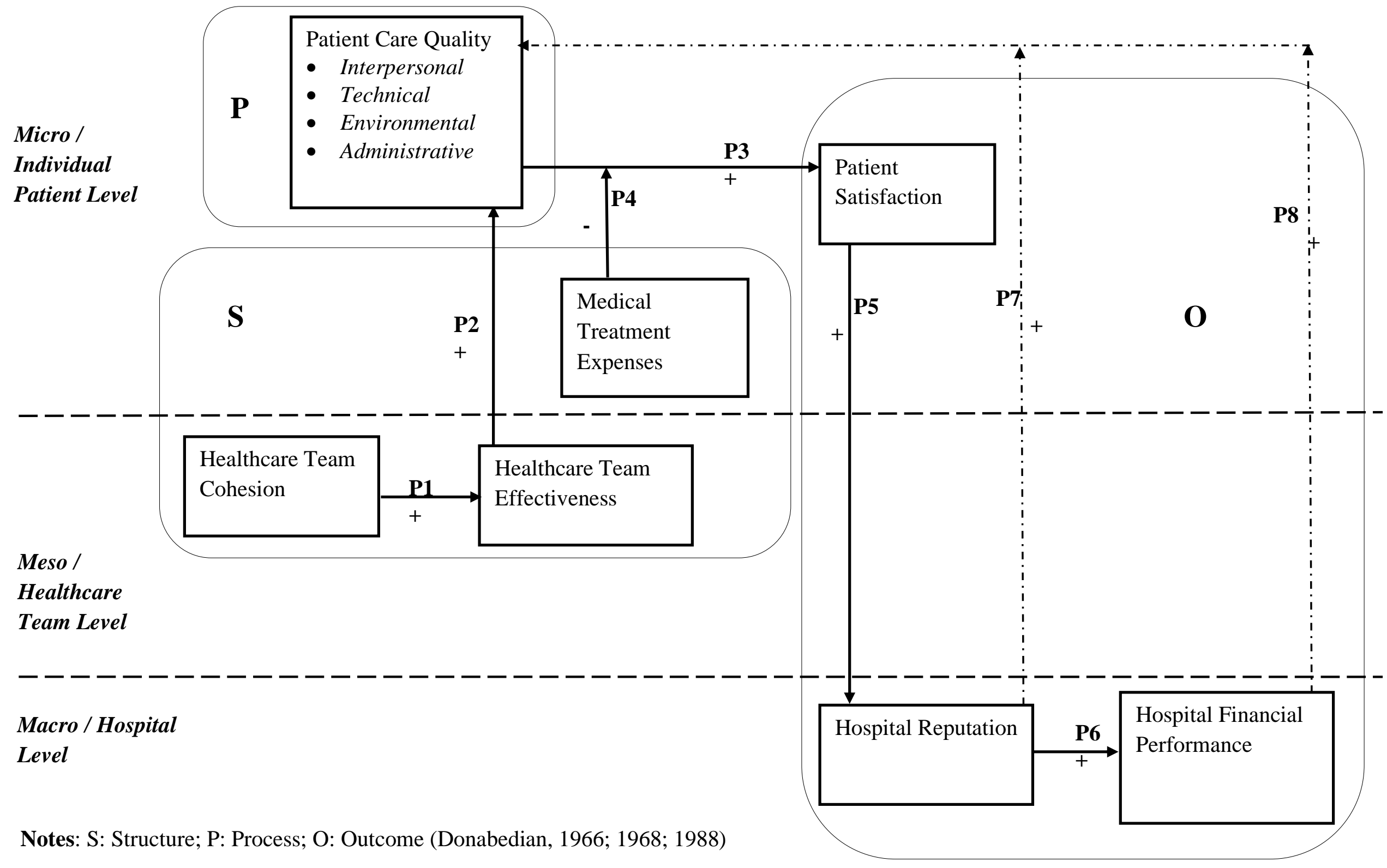

Figure 1 A multi-level healthcare quality framework 


\section{AN INTEGRATED MULTI-LEVEL HEALTHCARE QUALITY MEASUREMENT FRAMEWORK}

Using the dominant paradigm from healthcare and marketing literature-Structure-Process-Outcomes (SPO) (Donabedian, 1966, 1968, 1988) we place our conceptual framework in perspective. In so doing we extend Donabedian's SPO paradigm to include different variables that spread across three levels of measurement (micro, meso and macro) and also include long-term cyclical effects. Per the SPO guidelines (Donabedian, 1988) the three variableshealthcare team cohesion, healthcare team effectiveness and medical treatment expenses would be considered as structure (because these variables are concerned with the context of care); PCQ as the process (because it denotes the transactions between patients and healthcare providers during the medical treatment and even beyond); and the three variables - patient satisfaction, hospital reputation, and hospital financial performance as the outcomes (because they refer to the effects of healthcare on the health status of patients and populations). Our conceptual framework is depicted in Figure 1. Next, we offer eight propositions that detail our expected relationships in the following sections.

\subsection{Healthcare Team Cohesion, Team Effectiveness and Patient Care Quality}

Lemieux-Charles and McGuire (2006) note that the type and diversity of clinical expertise involved in team decision making is related to improvements in patient care and organizational effectiveness. Collaboration, conflict resolution, participation and cohesion influence staff satisfaction and perceived team effectiveness. The context in which teams are embedded also needs to be considered. Firms need to have cohesive cross-functional teams in order to implement quality practices across the organization because cohesive groups have a high degree of group identity and commitment to the group's tasks (Govers, 2001; Wang et al., 2006). This premise is based upon QM philosophy (Feigenbaum, 1961) which emphasizes team work as a required characteristic to achieve good employee relations, which in turn is required to implement quality practices. Effective healthcare teams perform their routine tasks little differently than other teams and learn quickly from each other. Small surgical team members are able to quickly learn from each other due to workload sharing and team helping, especially when the task complexity is very high (Vashdi et al., 2013).

Extant healthcare literature highlights that in order to be effective teams use physician empathy (Kim et al., 2004) and nurse emotional involvement (McQueen, 2000) to positively influence the interpersonal relationships that the healthcare teams are able to establish with their patients. Effective healthcare teams take an active interest in their patients' medical condition, empathize with their suffering (Roark and Sharah, 1989), communicate clearly to the patient and his/her kin about their medical condition and unanimously work toward their quicker cure, which would result in better interpersonal quality of patient care (DeeterSchmelz and Kennedy, 2003).

Adequate and timely access to and use of up-to-date patient health information can enhance the technical quality of patient care by providing physicians and nurses the correct up-to-date information on the patient's health (Chen et al., 2009; Jha et al., 2009). Effective healthcare teams can use electronic health records (EHR) and other hospital medical information systems to have all patient information readily available to the physicians for decision-making and nurses for support (Graetz et al., 2014).

All physical elements of a patient's environment such as the hospital bed, clothes and equipment must be clean and disinfected (Aiken et al., 2008). Hand hygiene prevents infection among patients and others (Pittet et al., 2000). Effective healthcare teams meticulously follow all hospital procedures, take all necessary precautions related to hygiene, ensure that all physical elements of the hospital including the beds and other medical and surgical equipment are thoroughly cleaned and disinfected before use on any patient (Carling et al., 2008). Further, effective healthcare teams interact with housekeeping to ensure that the hospital wards are organized, clean and aesthetically pleasing (Mathur, 2014). These steps would result in better environmental quality.

Hospital administration departments like billing and reception typically tend to work with a silo mentality in isolation (Bokar and Perry, 2007; Conway, 1997) oblivious to the fact that all departments need to support the healthcare teams in their effort to provide quality patient care. Effective healthcare teams can interact closely with the hospital's administrative units (White and Whitman, 2006) to ensure that information is provided timely to the patients or their next of kin. Because administrative quality is a key element of patient care (Grumbach and Bodenheimer, 2004), effective healthcare teams can frame a few preliminary procedures of their own to educate patients on simple administrative steps such as scheduling hospital visit appointments, providing food and dietary information related to the sickness to the patient or to their kin, explaining healthcare decision making processes to patients, and when required, interacting with the hospital's administrative departments on behalf of patients (Grumbach and Bodenheimer, 2004).

Based on the above discussions, we suggest that to become effective, members of a healthcare team need to work cohesively to avoid medical errors, check schedules and room/equipment availability in advance of patients' medical procedures, take steps to prevent infections in hospitals and keep the patients' care at the forefront of their decision making (Grumbach and Bodenheimer, 2004). All these activities would make patient care safer and more accurate, improving its overall quality. Therefore, drawing from tenets of QM philosophy we propose:

Proposition 1: Healthcare team cohesion is positively associated with healthcare team effectiveness.

Proposition 2: Healthcare team effectiveness is positively associated with patient care quality.

\subsection{Patient Care Quality and Satisfaction}

In healthcare, like in marketing of other services patient satisfaction is considered the defining core of all medical treatment philosophy that truly cares for the patient's medical treatment and thereby their cure from the ailment. Improved patient satisfaction is supposed to enhance the 
patient experience and ultimately improve the treatment outcomes (Rickert, 2014). Jha and colleagues (2008) empirically demonstrate that that the hospitals could simultaneously provide both high quality of care and a good patient experience by having high levels of nurse-staffing. They also found moderately high levels of satisfaction with care with a high degree of correlation among the measures of patients' experiences. A few articles have pointed out that quality of the medical treatment may not be directly related to patient satisfaction (Brookes and Fenton, 2014; Fenton et $a l ., 2012$ ) and hospitals and physicians may try to satisfy the patient but they not really provide the accurate medical treatment necessary to cure the patient of his/her ailment (Robbins, 2015). Nevertheless, there is a body of empirical research evidence in healthcare and marketing literature demonstrating that patient care quality is positively associated with patient satisfaction (Chang et al., 2009; Dagger et al., 2007). We therefore posit:

Proposition 3: Patient care quality is positively associated with patient satisfaction.

\subsection{Moderating Role of Medical Treatment Expenses}

The business press keeps publishing rich stories depicting how medical treatment in the U.S. is becoming prohibitively expensive for the common man with many even speculating and offering suggestions on how to control the spiraling healthcare costs (Arora et al., 2015; Robert Wood Johnson Foundation, 2016). In the U.S. healthcare system, there are many entities which affect the medical treatment costs and the patient's medical expenses. Apart from a hospital's supply chain partners who determine the cost of each item, the Group Purchasing Organizations (GPO) determines their group member hospital purchases and as a result, yields too much power over individual hospital costs of medical treatment (Handfield, 2010). Group Purchasing Organizations (GPO) such as Amerinet, Consorta, Novation, Premier and MedAssets were created to leverage the purchasing power of hospitals in obtaining discounts from vendors. A 2005 study for the Health Industry Group Purchasing Association (HIGPA) reported that 72$80 \%$ of every acute healthcare dollar is acquired through GPOs (Hu \& Schwarz, 2011). As already noted, healthcare insurance companies act as the intermediary between the hospital and the patient and have the final say in determining the actual medical expenses that a patient has to ultimately pay.

Dranove and Stanley (1995) suggested that firms may reduce costs by eliminating redundancies and reducing their administrative costs such as those evident in integration of many hospitals into a hospital system, which also help individual hospitals share the best quality practices. Venkataraman (2015) empirically established most hospitals may need to trade-off between keeping costs low and providing high quality care to patients. It is known that if the costs associated with the medical treatment are astronomically high, patients may have a very hard time paying off these high medical expenses (Mangan, 2013) and are likely to be extremely dissatisfied, even though their ailment or sickness may have been cured. On the other hand, if the costs are manageable for the patient, the patient is more than likely to be satisfied to some extent, even if the patient is not fully cured of his/her ailment/disease and may need to come back to the hospital. Therefore, we suggest a negative moderating role for treatment costs:

Proposition 4: Medical treatment expenses negatively moderate the relationship between patient care quality and patient satisfaction such that higher medical treatment expenses decrease patient satisfaction, and vice versa.

\subsection{Patient Satisfaction and Hospital Reputation}

Each individual physician's reputation adds to the hospital's reputation which is very important for the hospital's long-term sustenance (Angell, 1901). The professional culture of medicine poses unique challenges for building and sustaining a safety culture in a hospital because a strong emphasis on individual accountability for error often results in "blaming and shaming" individuals for the errors that have occurred under their supervision (Carroll and Quijada, 2004). Thus, many times doctors, nurses and hospital administrators may remain silent due to the fear of losing their individual and collective hospital reputations, even when they recognize unsafe conditions (Sutcliffe et al., 2004). The quality of supplies delivered by the suppliers to a firm (Gunasekaran et al., 2004; Larson, 1994) is also important for a long term relationship because poor quality products and services could lead to rework for the supplier and loss of reputation and image for the hospital. Inferior quality products used in healthcare may also cause patient harm.

Healthcare research has demonstrated that the social reputation of hospitals and the perceptions of patients/kin of patient are correlated (Mira et al., 2014). Dissatisfied patients, like many other dissatisfied service customers, are known to spread bad word of mouth and vent their anger on social media like Yelp (Ranard et al., 2016). Thus, in today's modern world if patients are not satisfied with any aspect of their medical treatment, they are likely to spread bad word of mouth and complain using one or more platforms like social media and/or Joint Commission or their state's quality improvement organizations (QIO) (Snyder and Anderson, 2005), which could harm the individual physician's reputation and in turn, negatively affect the hospital reputation. On the positive side, satisfied patients are likely to spread the good word about the physicians, the hospital and its facilities and the quality of care offered therein. Hence, we posit:

Proposition 5: Patient satisfaction is positively associated hospital reputation.

\subsection{Hospital Reputation, Financial Performance, and Effects on Patient Care Quality}

Social reputation of hospitals is known to be correlated with patient safety perceptions of patients/kin (Mira et al., 2014). With preventable hospital errors now being a key focus area of healthcare reform, nurses have a very important contribution to ensure patient safety, quality of care, and the hospital's financial performance (Kohlbrenner et al., 2011). Improving patient satisfaction can have a direct impact on the hospital's reputation, and financial results (Hall, 2008). A 
recent study from the Deloitte Center for Health SolutionsThe Value of Patient Experience-reports that "improving the patient experience can help a hospital improve its financial performance by strengthening customer loyalty, building reputation and brand, and boosting utilization of hospital services through increased referrals to family and friends" (Betts, 2017). For several years now, the Centers for Medicare and Medicaid Services (CMS) has been using patient-reported experience scores, which affect a hospital's reputation, to reimburse and reward or penalize hospitals through Medicare's Value Based Purchasing program. Many hospitals have started using the Hospital Consumer Assessment of Healthcare Providers and Systems (HCAHPS) survey scores to justify their greater investment in improving the patient experience during their hospital stay (Mehta, 2015). Therefore, based on the above discussions, we propose:

Proposition 6: Hospital reputation is positively associated with hospital financial performance.

For quality initiatives to be ingrained in the organization, teams need active support from their leaders. Research has demonstrated empirical support for the relationship between financial performance and quality of care (Bazzoli et al., 2008) but was not as strong as suggested in previous research. QM philosophy (Feigenbaum, 1961) suggests that senior management needs to provide resources like funds and trained personnel to make the work environment conducive for teams to work. If hospitals do well financially, its leaders can not only better sell the ongoing quality story externally to all stakeholders, but also have a better chance of supporting the quality improvement efforts of all physicians, nurses and staff by improving resource allocations to the departments as necessary. Hospital facilities expansion, internal technology infrastructure upgrades, which can help in process improvements as well as increase in nurse and staff headcount and thereby more manageable work shifts become possible with more funds available to hospital leadership.

On a similar note, hospitals having a good reputation (e.g., those being featured in the America's Best Hospitals each year) are generally managed by reputed doctors (Stoller et al., 2016) and are also able to draw other reputed/famous physicians (Rizo et al., 2002). This in turn, helps improve the patient experience at the hospital (Pope, 2009) thereby bringing in a steady stream of patients as well. Hospitals of repute put significant collective effort and resources to maintaining high PCQ standards in order to maintain their high public rankings and good patient ratings (Luxford et al., 2011). Therefore, based on the above long-term phenomena our final two propositions suggest:

Proposition 7: Hospital reputation is positively associated with patient care quality.

Proposition 8: Hospital financial performance is positively associated with patient care quality.

\section{DISCUSSION AND CONCLUSION}

In this paper, we proposed a conceptual framework for measuring PCQ, along with its antecedents and outcomes.
We adopted a multi-level approach to measuring PCQ and described the role of healthcare team-related antecedentshealthcare team cohesion and healthcare team effectiveness that affect individual patient-centric variables-PCQ, medical treatment expenses and patient satisfaction, which ultimately affect the hospital-level outcomes-hospital reputation and hospital financial performance. We also suggested a moderating effect of medical treatment expenses on the PCQ-patient satisfaction relationship and proposed a feedback loop of hospital reputation and hospital performance affecting PCQ, over time. Essentially, we traced the full quality flow chain in a typical U.S. hospital. The conceptual framework proposed here has useful implications for both research and medical practice at hospitals.

Our framework recognizes the important role of teamlevel antecedents affecting individual patient-centric variables, which finally affect the hospital-level outcomes. Essentially, it acknowledges the complexity of the U.S. healthcare system with so many entities and why/how so many changes being made are yet to lower the cost of medical treatments or improve PCQ, across the board. The research also suggests an important moderating role for medical treatment expenses that are skyrocketing every year on the PCQ-satisfaction relationship.

Our conceptual framework is only a first step and should spur multi-level empirical healthcare research on PCQ. A planned empirical measurement of the framework should reveal interesting insights. All variables could be measured using perceptual measures and supplemented with objective secondary data on medical treatment costs and hospital financial performance. An extensive online (Qualtrics) national survey campaign is planned that will randomly select both patients from U.S. hospitals, as well U.S. hospital administrators, including members of healthcare teams such as physicians, nurses and staff. Since this study is essentially longitudinal we propose two sets of measurements each six month apart targeting the same hospitals in both survey waves.

The model in its entirety, or a few specific relationships may be tested using available measures from literature and longitudinal data gathering. PCQ may be measured using modified scales from literature (Dagger et al., 2007; Gill \& White, 2009). Patient satisfaction may be measured using scales based on Ware and colleagues (1983). The patient's perceptions of the medical expenses can be obtained using scales adapted from Maratt et al. (2015) and hard secondary data available on average medical expenses for specific diseases, could be used to supplement the perceptual measures. Healthcare team cohesion can be measured using a modified version of the Team Development Measure (Stock et al., 2013). Healthcare team effectiveness may be measured using scales adapted from Poulton \& West (1993, 1999). Hospital reputation can be tapped using a scale adapted from Mira et al. (2014). Hospital financial performance can be measured using scale adapted from Cleverley and Rohleder (1985). Again, secondary data on hospital financial performance can be obtained from public databases such as the American Hospital Directory, Mergent Online and other strategic management databases to supplement the perceptual measures.

An empirical test of our conceptual framework will help clear the air on some of the controversial relationships 
among variables such as the relationship between healthcare costs and PCQ or the relationship between PCQ and patient satisfaction that scholars have argued upon in the literature. The multilevel nature of the framework suggests that future empirical studies may need to use nested design, such as one involving dyads of patients and healthcare team from more than one U.S. hospital.

Our conceptual framework has several implications for medical practitioners and U.S. hospitals. First, the framework amply demonstrates that healthcare team cohesion and effectiveness are the most important antecedents to high quality of patient care and should either encourage or coax all medical practitioners including physicians, nurses and other hospital staff to work together, seamlessly for the delivering the highest PCQ. In this context, many hospitals may need to organize special focused trainings and live patient treatment sessions for its senior physicians and nurses, imploring them to work together and not treat patients' medical treatment as a turf fight.

Second, U.S. hospitals could provide high quality of admitted patient care if they give importance to all the variables and the interrelationships described in the research model which would reduce medical errors and help them operate at the highest level of efficiency (Byrnes, 2004; Shih et al., 2009). Hospitals delivering high quality of admitted patient care is very crucial for all stakeholders because it could improve hospitals' financials, benefit all entities in the supply chain, and help the admitted patients directly through better and more responsive medical care that cures them of their ailments quicker and at lower cost (Lee et al., 2011).

Third, hospital leaders need to refocus their energies on the efficient and effective use of technology throughout their hospital to share ideas and most importantly, ensure that their healthcare team members have the most accurate and up-todate information on patients. Although many U.S. hospitals are already using patient information systems, it tends not to be uniform across all hospitals and departments (Schroeder, 2015). Even today, not all U.S. hospital systems interact with each other to communicate the most up-to-date information (Whitney, 2015).

\subsection{Limitations and Future Directions}

Because we focused on PCQ measurement in the U.S. hospital ecosystem, our conceptual framework is applicable for U.S. healthcare system and caution must be exercised before extending it to other countries. Although all the variables in the framework and their proposed associative relationships would still hold in most other countries, the role of government-provided insurance is very important in countries like the U.K., Canada and Australia and even in Mexico and India and insurance provision may affect some of the hypothesized relationships. Further, in empirical measurement, summated scores may need to be calculated to address the difference in levels issue among the different variables, thereby losing some of the richness of the individual patient level data. Nevertheless, we do not foresee any major issue in empirical measurement as previous studies have successfully addressed the different levelsissue.

In this paper, we used the prominent paradigm in healthcare literature-the SPO guidelines (Donabedian,
$1966,1968,1988)$ to place our research in perspective with the extant marketing and healthcare literature. Aligning our framework with this paradigm helped us build a cyclical process model that investigates the causes and outcomes of PCQ in the U.S. healthcare system. In so doing, we could fully trace the quality chain in a typical U.S. hospital. We also extended the SPO paradigm by including variables spread across different levels of measurement and different periods of time to include cyclical effects. Future research could empirically test the conceptual framework offered herein.

Another avenue for future research is to investigate how team-level variables end up influencing the primary individual-level variable of interest, i.e., PCQ and finally how they influence the hospital-level outcomes. Future research could also focus on finding out the possible service failure points to make them fail-safe and map out complete service blueprints for each major disease/ailment at a typical U.S. hospital. Such empirically tested service blueprints could be used by hospital departments to improve the service delivery and efficiency.

To the best of our knowledge, the proposed conceptual framework is the first to link all antecedents to and outcomes of PCQ in the U.S. healthcare ecosystem. The empirical test of the longitudinal link between hospital financial performance and PCQ should help clear the ongoing debate on how PCQ actually helps hospitals achieve a sustainable competitive advantage, like in other service industries. After all, for people to receive the correct healthcare at the minimum cost, hospitals must be able to thrive.

\section{REFERENCES}

Aiken, L.H., Clarke, S.P., and Sloane, D.M. (2008). Effects of hospital care environment on patient mortality and nurse outcomes. Journal of Nursing Administration 38(5), pp. 223 $-229$.

Alexander, J.A., Weiner, B.J., and Griffith, J. (2006). Quality Improvement and Hospital Financial Performance. Journal of Organizational Behavior 27(7), pp. 1003 - 1029.

Angell, E.B. (1901). The Modern Hospital: Its Value to the Patient and to the Physician. The American Journal of Nursing 1(10), pp. $703-712$.

Arora, V., Moriates, C., and Shah, N. (2015). The Challenge of Understanding Health Care Costs and Charges. AMA Journal of Ethics 17(11), pp. 1046.

Bazzoli, G.J., Chen, H.-F., Zhao, M., and Lindrooth, R.C. (2008). Hospital Financial Condition and the Quality of Patient Care. Health Economics 17(8), pp. 977 - 995.

Beal, D.J., Cohen, R.R., Burke, M.J., and McLendon, C.L. (2003). Cohesion and Performance in Groups: A Meta-Analytic Clarification of Construct Relations. Journal of Applied Psychology 88(6), pp. 989 - 1004.

Becker, S., and Gamble, M. (2015), The 8 biggest healthcare issues in 2015 so far, available at: http://www.beckershospitalreview.com/hospitalmanagement-administration/the-8-biggest-healthcare-issuesin-2015-so-far.html (accessed 6 February 2017).

Bender, B.G., and Rand, C. (2004). Medication non-adherence and asthma treatment cost. Current Opinion in Allergy and Clinical Immunology 4(3), pp. 191-195.

Betts, D. (2017), Value of patient experience, Deloitte United States, available at: https://www2.deloitte.com/us/en/pages/life-sciences-andhealth-care/articles/hospitals-patient-experience.html (accessed 23 April 2017). 
Bokar, V., and Perry, D.G. (2007). Different roles, same goal: risk and quality management partnering for patient safety. By the ASHRM Monographs Task Force. Journal of Healthcare Risk Management: The Journal of the American Society for Healthcare Risk Management 27(2), pp. 17 - 23, 25.

Boyer, K.K., and Pronovost, P. (2010). What medicine can teach operations: What operations can teach medicine. Journal of Operations Management, pp. 367 - 371.

Brookes, L., and Fenton, J.J. (2014), Patient Satisfaction and Quality of Care: Are They Linked?, Medscape, available at: http://www.medscape.com/viewarticle/826280 (accessed 22 April 2017).

Buljac-Samardzic, M., Dekker-van Doorn, C.M., van Wijngaarden, J.D.H., and van Wijk, K.P. (2010). Interventions to improve team effectiveness: A systematic review. Health Policy 94(3), pp. $183-195$.

Byrnes, J. (2004), Fixing the Healthcare Supply Chain, Harvard Business School Working Knowledge, Harvard Business School, available at: http://hbswk.hbs.edu/archive/4036.html.

Carling, P.C., Parry, M.F., and Von Beheren, S.M. (2008). Identifying Opportunities to Enhance Environmental Cleaning in 23 Acute Care Hospitals. Infection Control and Hospital Epidemiology 29(1), pp. 1 - 7.

Carroll, J.S. and Quijada, M.A. (2004), Redirecting traditional professional values to support safety: changing organisational culture in health care. Quality \& Safety in Health Care 13, pp. $16-21$.

Carron, A.V., and Brawley, L.R. (2000). Cohesion: Conceptual and Measurement Issues. Small Group Research 31(1), pp. $89-$ 106.

Chandrasekaran, A., Senot, C., and Boyer, K.K. (2012). Process Management Impact on Clinical and Experiential Quality: Managing Tensions Between Safe and Patient-Centered Healthcare. M\&Som-Manufacturing \& Service Operations Management 14(4), pp. $548-566$.

Chang, W.Y., Ma, J.C., Chiu, H.T., Lin, K.C., and Lee, P.H. (2009). Job satisfaction and perceptions of quality of patient care, collaboration and teamwork in acute care hospitals. Journal of Advanced Nursing 65(9), pp. 1946 - 1955.

Chen, C., Garrido, T., Chock, D., Okawa, G., and Liang, L. (2009). The Kaiser Permanente Electronic Health Record: Transforming And Streamlining Modalities Of Care. Health Affairs 28(2), pp. 323 - 333.

Cleverley, W.O., and Rohleder, H. (1985). Unique dimensions of financial analysis service ratios. Topics in Health Care Financing 11(4), pp. $81-88$.

CMS. (2016), Quality Measures - Centers for Medicare \& Medicaid Services, Quality Measures, available at: https://www.cms.gov/Medicare/Quality-Initiatives-PatientAssessment-

Instruments/QualityMeasures/index.html?redirect=/Quality Measures/ (accessed 13 May 2017).

Cohen, S.G., and Bailey, D.E. (1997). What makes teams work: Group effectiveness research from the shop floor to the executive suite. Journal of Management 23(3), pp. 239-290.

Conway, J.B. (1997). Breaking down professional silos: the potential of integrated management, Radiology Management 19(3), pp. $30-34$.

Dagger, T.S., Sweeney, J.C., and Johnson, L.W. (2007). A Hierarchical Model of Health Service Quality: Scale Development and Investigation of an Integrated Model. Journal of Service Research 10(2), pp. 123 - 142.

Davis, K., Stremikis, K., Squires, D., and Schoen, C. (2014), Mirror, Mirror on the Wall, 2014 Update: How the U.S. Health Care System Compares Internationally, available at: http://www.commonwealthfund.org/publications/fundreports/2014/jun/mirror-mirror (accessed 24 April 2017).

Deeter-Schmelz, D.R., and Kennedy, K.N. (2003). Patient care teams and customer satisfaction: the role of team cohesion. Journal of Services Marketing 17(6/7), pp. 666 - 684.
Delva, D., Jamieson, M., and Lemieux, M. (2008). Team effectiveness in academic primary health care teams. Journal of Interprofessional Care 22(6), pp. 598-611.

Deming, W.E. (1986), Out of the Crisis, Massachusetts Institute of Technology Press, Cambridge, MA.

DiMatteo, M.R., Lepper, H.S., and Croghan, T.W. (2000). Depression Is a Risk Factor for Noncompliance With Medical Treatment: Meta-analysis of the Effects of Anxiety and Depression on Patient Adherence. Archives of Internal Medicine 160(14), pp. $2101-2107$.

Donabedian, A. (1966). Evaluating the quality of medical care. Milbank Memorial Fund Quarterly 44(Suppl), pp. 166 - 206.

Donabedian, A. (1968). Promoting Quality through Evaluating the Process of Patient Care. Medical Care 6(3), pp. 181- 202.

Donabedian, A. (1988). The quality of care. How can it be assessed?. JAMA 260(12), pp. 1743 - 1748.

Douglas, T.J., and Ryman, J.A. (2003). Understanding competitive advantage in the general hospital industry: evaluating strategic competencies. Strategic Management Journal 24(4), pp. $333-347$.

Dranove, D., and Shanley, M. (1995). Cost Reductions or Reputation Enhancement as Motives for Mergers: The Logic of Multihospital Systems. Strategic Management Journal $16(1)$, pp. $55-74$.

Feigenbaum, A.V. (1961), Total Quality Control, McGraw-Hill, New York.

Fenton, J.J., Jerant, A.F., Bertakis, K.D., and Franks, P. (2012). The cost of satisfaction: a national study of patient satisfaction, health care utilization, expenditures, and mortality. Archives of Internal Medicine 172(5), pp. 405 - 411.

Gill, L., and White, L. (2009). A critical review of patient satisfaction. Leadership in Health Services 22(1), pp. 8 - 19.

Goes, J.B., and Zhan, C. (1995). The effects of hospital-physician integration strategies on hospital financial performance. Health Services Research 30(4), pp. 507 - 530.

Goodman, P.S. (1986), Impact of task and technology on group performance, in P.S. Goodman \& Associates (Ed.). Designing Effective Work Groups, Jossey-Bass, San Francisco, CA.

Govers, C.P.M. (2001). QFD not just a tool but a way of quality management. International Journal of Production Economics 69(2), pp. $151-159$.

Graetz, I., Reed, M., Shortell, S.M., Rundall, T.G., Bellows, J., and Hsu, J. (2014). The Association between EHRs and Care Coordination Varies by Team Cohesion. Health Services Research 49(1pt2), pp. $438-452$.

Grumbach, K., and Bodenheimer, T. (2004). Can health care teams improve primary care practice?. JAMA 291(10), pp. 1246 1251.

Gunasekaran, A., Patel, C., and McGaughey, R.E. (2004). A framework for supply chain performance measurement. International Journal of Production Economics 87(3), pp. $333-347$.

Hall, M.F. (2008). Looking to improve financial results? Start by listening to patients. Healthcare Financial Management: Journal of the Healthcare Financial Management Association 62(10), pp. $76-80$.

Hall, R.H. (1980). Effectiveness Theory and Organizational Effectiveness. The Journal of Applied Behavioral Science 16(4), pp. $536-545$.

Handfield, R. (2010). Non-Healthcare Supply Chain Tools for the Healthcare Problem, available at: http://scm.ncsu.edu/blog/2010/06/08/non-healthcare-supplychain-tools-for-the-healthcare-problem/\#comments

Hibbard, J.H., Stockard, J., and Tusler, M. (2005). Hospital performance reports: impact on quality, market share, and reputation. Health Affairs 24(4), pp. $1150-1160$.

Huckman, R.S., and Zinner, D.E. (2008). Does focus improve operational performance? Lessons from the management of clinical trials. Strategic Management Journal 29(2), pp. 173 
-193 .

Jha, A.K., DesRoches, C.M., Campbell, E.G., Donelan, K., Rao, S.R., Ferris, T.G., Shields, A., et al. (2009). Use of Electronic Health Records in U. S. Hospitals. New England Journal of Medicine 360(16), pp. 1628 - 1638 .

Jha, A.K., Orav, E.J., Zheng, J., and Epstein, A.M. (2008). Patients' Perception of Hospital Care in the United States. New England Journal of Medicine (359), pp. 1921 - 1931.

Jolley, R.J., Lorenzetti, D.L., Manalili, K., Lu, M., Quan, H., and Santana, M.J. (2017). Protocol for a scoping review study to identify and classify patient-centred quality indicators. $B M J$ Open 7(1), pp. e013632.

Kaissi, A.A., and Begun, J.W. (2008). Strategic planning processes and hospital financial performance. Journal of Healthcare Management / American College of Healthcare Executives 53(3), pp. 197 - 208; discussion 208-209.

Katzenbach, J.R., and Smith, D.K. (2013), The Wisdom of Teams: Creating the High-Performance Organization, Harvard Business Review Press, Brighton, MA.

Kaynak, H. (2003). The relationship between total quality management practices and their effects on firm performance. Journal of Operations Management 21(4), pp. 405.

Kaynak, H., and Hartley, J.L. (2008), A replication and extension of quality management into the supply chain. Journal of Operations Management 26(4), pp. 468 - 489.

Kim, S.S., Kaplowitz, S., and Johnston, M.V. (2004). The Effects of Physician Empathy on Patient Satisfaction and Compliance. Evaluation \& the Health Professions 27(3), pp. $237-251$.

Kohlbrenner, J., Whitelaw, G., and Cannaday, D. (2011). Nurses critical to quality, safety, and now financial performance. Journal of Nursing Administration 41(3), pp. 122 - 128.

Kohn, L.T., Corrigan, J.M. and Donaldson, M.S. (1999), To Err Is Human: Building a Safer Health System, The National Academies Press, Washington, DC.

Kujala, J. and Lillrank, P. (2004). ASQ: Total Quality Management as a Cultural Phenomenon, available at: http://asq.org/qic/display-item/?item=19683 (accessed 24 April 2017).

Larson, P.D. (1994). Buyer-Supplier Co-operation, Product Quality and Total Costs. International Journal of Physical Distribution \& Logistics Management 24(6), pp. 4.

Laschinger, H.K.S., and Leiter, M.P. (2006). The Impact of Nursing Work Environments on Patient Safety Outcomes: The Mediating Role of Burnout Engagement. Journal of Nursing Administration 36(5), pp. 259 - 267.

Lee, S.M., Lee, D.H., and Schniederjans, M.J. (2011). Supply Chain Innovation and Organizational Performance in the Health Care Industry. International Journal of Operations \& Production Management 31(11), pp. 1193 - 1214.

Lemieux-Charles, L., and McGuire, W.L. (2006). What Do We Know about Health Care Team Effectiveness? A Review of the Literature. Medical Care Research and Review 63(3), pp. $263-300$.

Lott, A.J., and Lott, B.E. (1965). Group cohesiveness as interpersonal attraction: A review of relationships with antecedent and consequent variables. Psychological Bulletin 64(4), pp. $259-309$.

Luxford, K., Safran, D.G., and Delbanco, T. (2011). Promoting patient-centered care: a qualitative study of facilitators and barriers in healthcare organizations with a reputation for improving the patient experience. International Journal for Quality in Health Care 23(5), pp. 510 - 515.

Mangan, D. (2013), Medical Bills Are the Biggest Cause of US Bankruptcies: Study, available at: http://www.cnbc.com/id/100840148 (accessed 24 April 2017).

Maratt, J.D., Gagnier, J.J., Gombera, M.M., Reske, S.E., Hallstrom, B.R., and Urquhart, A.G. (2015). Patients' Perceptions of the Costs of Total Hip and Knee Arthroplasty. The American
Journal of Orthopedics 44(5), pp. E135-141.

Mark, T.L., Evans, W.N., Schur, C.L., and Guterman, S. (1998). Hospital-Physician Arrangements and Hospital Financial Performance. Medical Care 36(1), pp. 67 - 78.

Mathur, P. (2014), Role of Hospital Housekeeping and Materials Management Including Disinfection and Waste Management, in C. Wattal, and N. Khardori, (Ed.). Hospital Infection Prevention, Springer India), pp. 81 - 89.

McColl-Kennedy, J.R., Snyder, H., Elg, M., Witell, L., Helkkula, A., Hogan, S.J., and Anderson, L. (2017). The changing role of the health care customer: review, synthesis and research agenda. Journal of Service Management 28(1), pp. 2 - 33.

McFadden, K.L., Henagan, S.C., and Gowen Iii, C.R. (2009). The patient safety chain: Transformational leadership's effect on patient safety culture, initiatives, and outcomes. Journal of Operations Management 27(5), pp. 390 - 404.

McGregor, D. (1987), The Human Side of Enterprise, McGrawHill, New York.

McQueen, A. (2000). Nurse - patient relationships and partnership in hospital care. Journal of Clinical Nursing 9(5), pp. $723-$ 731

Mehta, S.J. (2015). Patient Satisfaction Reporting and Its Implications for Patient Care. AMA Journal of Ethics 17(7), pp. 616.

de Mheen, M.P.J., Dijs-Elsinga, J., Otten, W., Versluijs, M., Smeets, H.J., van der Made, W.J., Vree, R., et al. (2010). The importance of experienced adverse outcomes on patients future choice of a hospital for surgery. Quality \& Safety in Health Care 19(6), pp. e16.

Mira, J.J., Lorenzo, S., and Navarro, I. (2014). Hospital reputation and perceptions of patient safety. Medical Principles and Practice: International Journal of the Kuwait University, Health Science Centre 23(1), pp. 92 - 94.

Mosadeghrad, A.M. (2013). Healthcare service quality: towards a broad definition. International Journal of Health Care Quality Assurance 26(3), pp. 203 - 219.

Mullen, B., and Copper, C. (1994). The relation between group cohesiveness and performance: An integration. Psychological Bulletin 115(2), pp. 210.

Nelson, C.W., and Niederberger, J. (1990). Patient satisfaction surveys: An opportunity for Total quality improvement. Hospital \& Health Services Administration 35(3), pp. 409.

Pittet, D., Hugonnet, S., Harbarth, S., Mourouga, P., Sauvan, V., Touveneau, S., Perneger, T.V., et al. (2000). Effectiveness of a hospital-wide programme to improve compliance with hand hygiene. Lancet 356(9238), pp. $1307-1312$.

Pope, D.G. (2009). Reacting to rankings: Evidence from 'America's Best Hospitals'. Journal of Health Economics 28(6), pp. $1154-1165$

Poulton, B.C., and West, M.A. (1993). Effective multidisciplinary teamwork in primary health care. Journal of Advanced Nursing 18(6), pp. $918-925$.

Poulton, B.C., and West, M.A. (1999). The determinants of effectiveness in primary health care teams. Journal of Interprofessional Care 13(1), pp. 7 - 18 .

Price, J., L., and Mueller, C.W. (1986), Handbook of Organizational Measurement, Pitman, available at: http://oss.sagepub.com/content/9/1/134.short.

Pronovost, P.J., Miller, M.R., and Wachter, R.M. (2006). Tracking progress in patient safety - An elusive target. Jama-Journal of the American Medical Association 296(6), pp. 696 - 699.

Queenan, C.C., Angst, C.M., and Devaraj, S. (2011). Doctors' orders - - If they're electronic, do they improve patient satisfaction? A complements/substitutes perspective. Journal of Operations Management 29(7-8), pp. 639-649.

Ranard, B.L., Werner, R.M., Antanavicius, T., Schwartz, H.A., Smith, R.J., Meisel, Z.F., Asch, D.A., et al. (2016). Yelp Reviews Of Hospital Care Can Supplement And Inform Traditional Surveys Of The Patient Experience Of Care. Health Affairs 35(4), pp. $697-705$. 
Rickert, J. (2014). Measuring Patient Satisfaction: A Bridge Between Patient And Physician Perceptions Of Care. Health Affairs, available at: http://healthaffairs.org/blog/2014/05/09/measuring-patientsatisfaction-a-bridge-between-patient-and-physicianperceptions-of-care/ (accessed 22 April 2017).

Rizo, C.A., Jadad, A.R., and Enkin, M. (2002). What's a good doctor and how do you make one?. BMJ: British Medical Journal 325(7366), pp. 711.

Roark, A.E., and Sharah, H.S. (1989). Factors Related To Group Cohesiveness. Small Group Research 20(1), pp. 62 - 69.

Robbins, A. (2015). The Problem With Satisfied Patients - The Atlantic, The Atlantic, available at: https://www.theatlantic.com/health/archive/2015/04/theproblem-with-satisfied-patients/390684/ (accessed 22 April 2017).

Robert Wood Johnson Foundation. (2016). How Price Transparency Can Control the Cost of Health Care, RWJF, available

https://www.rwjf.org/en/library/research/2016/03/howprice-transparency-controls-health-care-cost.html (accessed 3 January 2018).

Rogers, A.E., Hwang, W.-T., Scott, L.D., Aiken, L.H., and Dinges, D.F. (2004). The Working Hours Of Hospital Staff Nurses And Patient Safety. Health Affairs 23(4), pp. $202-212$.

Schroeder, M. (2015). Hospitals Are Moving (Slowly) to Electronic Medical Records, available at: http://health.usnews.com/health-news/besthospitals/articles/2015/10/15/hospitals-are-moving-slowlyto-electronic-medical-records.

Sehgal, A.R. (2010). The Role of Reputation in U.S. News: World Report's Rankings of the Top 50 American Hospitals. Annals of Internal Medicine 152(8), pp. 521.

Shih, S.C., Rivers, P.A., and Soya Hsu, H.Y. (2009). Strategic information technology alliances for effective health-care supply chain management. Health Services Management Research 22(3), pp. $140-150$.

Shipper, F., and White, C.S. (1983). Linking organizational effectiveness and environmental change. Long Range Planning 16(3), pp. $99-106$.

Shortell, S.M., Marsteller, J.A., Lin, M., Pearson, M.L., Wu, S.-Y., Mendel, P., Cretin, S., et al. (2004). The Role of Perceived Team Effectiveness in Improving Chronic Illness Care. Medical Care 42(11), pp. 1040 - 1048.

Singer, S., Meterko, M., Baker, L., Gaba, D., Falwell, A., and Rosen, A. (2007). Workforce Perceptions of Hospital Safety Culture: Development and Validation of the Patient Safety Climate in Healthcare Organizations Survey. Health Services Research 42(5), pp. 1999 - 2021.

Snyder, C., and Anderson, G. (2005). Do quality improvement organizations improve the quality of hospital care for medicare beneficiaries?. JAMA 293(23), pp. 2900 - 2907.

Sokol, M.C., McGuigan, K.A., Verbrugge, R.R., and Epstein, R.S.
(2005). Impact of Medication Adherence on Hospitalization Risk and Healthcare Cost. Medical Care 43(6), pp. 521-530.

Stock, R., Mahoney, E., and Carney, P.A. (2013). Measuring Team Development in Clinical Care Settings. Family Medicine 45(10), pp. $691-700$.

Stoller, J.K., Goodall, A. and Baker, A. (2016). Why The Best Hospitals Are Managed by Doctors. Harvard Business Review, 27 December, available at: https://hbr.org/2016/12/why-the-best-hospitals-aremanaged-by-doctors (accessed 4 January 2018).

Sundstrom, E., De Meuse, K.P., and Futrell, D. (1990). Work Teams: Applications and Effectiveness. The American Psychologist 45(2), pp. 120.

Sutcliffe, K.M., Lewton, E., and Rosenthal, M.M. (2004). Communication failures: An insidious contributor to medical mishaps. Academic Medicine 79(2), pp. 186 - 194.

Sweeney, J.C., Danaher, T.S., and McColl-Kennedy, J.R. (2015). Customer Effort in Value Cocreation Activities. Journal of Service Research 18(3), pp. 318 - 335.

Vashdi, D.R., Bamberger, P.A., and Erez, M. (2013). Can Surgical Teams Ever Learn? The Role of Coordination, Complexity, and Transitivity in Action Team Learning. Academy of Management Journal 56(4), pp. 945 - 971.

Venkataraman, S. (2015), Cost-Quality Tradeoff in Healthcare: Does it Affect Patient Experience? Quality Management Journal 22(3), pp. 38-45.

Voyce, J., Gouveia, M.J.B., Medinas, M.A., Santos, A.S., and Ferreira, R.F. (2015). A Donabedian Model of the Quality of Nursing Care From Nurses' Perspectives in a Portuguese Hospital: A Pilot Study. Journal of Nursing Measurement 23(3), pp. $474-484$.

Wang, E.T.G., Ying, T.-C., Jiang, J.J., and Klein, G. (2006). Group cohesion in organizational innovation: An empirical examination of ERP implementation. Information and Software Technology 48(4), pp. 235 - 244.

Ware, J.E., Snyder, M.K., Wright, W.R., and Davies, A.R. (1983) Defining and measuring patient satisfaction with medical care. Evaluation and Program Planning 6(3-4), pp. $247-$ 263.

White, K., and Whitman, P. (2006). Caught the 'Big One': How a Newly Integrated Home Health Billing Department, Working With a Coach, Used the FISH Philosophy to Catch Team Spirit. Home Health Care Management \& Practice 18(4), pp. $300-305$.

Whitney, E. (2015). Sharing Patient Records Is Still A Digital Dilemma For Doctors, Health Inc., available at: http://www.npr.org/sections/health-

shots/2015/03/06/388999602/sharing-patient-records-is-stilla-digital-dilemma-for-doctors.

Yukelson, D., Weinberg, R., and Jackson, A. (1984). A multidimensional group cohesion instrument for intercollegiate basketball teams, Journal of Sport Psychology 6(1), pp. $103-117$.

Dr. Subhajit Chakraborty is an Assistant Professor of Management and Decision Sciences in the E. Craig Wall Sr. College of Business Administration at Coastal Carolina University. He holds a Ph.D. in Business Administration from The University of Texas Rio Grande Valley. He has 11 years of practitioner experience in operations, supply chain and information technology domains with project management and expatriate experience and is a member of the American Society for Quality. His research interests include service quality along firms' supply chain, service design and lean healthcare operations. His research has been published in journals such as Quality Management Journal and Health Marketing Quarterly. 